\title{
Simple good approximations for the special elliptic functions in standard Fowler-Nordheim tunneling theory for a Schottky-Nordheim barrier
}

\author{
Richard G. Forbes ${ }^{\text {a) }}$ \\ Advanced Technology Institute (BB), School of Electronics and Physical Sciences, University of Surrey, \\ Guildford, Surrey GU2 7XH, United Kingdom
}

(Received 9 May 2006; accepted 3 August 2006; published online 15 September 2006)

\begin{abstract}
The discovery is reported of simple, good approximate formulas for special elliptic functions that appear in the standard theory of Fowler-Nordheim (FN) [Proc. R. Soc. London, Ser. A 119, 173 (1914)] tunneling through an image-rounded Schottky-Nordheim [W. Schottky, Z. Phys. 15, 872 (1923); L. W. Nordheim, Proc. R. Soc. London, Ser. A 121, 626 (1928)] barrier and in the standard FN equation. The FN-exponent correction factor $v$ can be written as $v(y) \approx 1-y^{2}+(1 / 3) y^{2} \ln y$, where $y$ is the Nordheim parameter. This formula has a respectable mathematical basis, predicts exact values of $v(y)$ to within $0.33 \%$ in $0 \leqslant y \leqslant 1$, and can be rewritten to give (after nearly 80 years) a simple, reliable algebraic formula for the explicit dependence of $v$ on barrier field. Significant consequences are expected. (C) 2006 American Institute of Physics. [DOI: 10.1063/1.2354582]
\end{abstract}

This letter reports good approximations for the special elliptic functions that appear in the standard theory of Fowler-Nordheim tunneling and cold field electron emission (CFE). These processes have wide technical relevance, in particular, (a) as the mechanism by which high-voltage breakdown is often initiated, (b) as an electron-transfer mechanism in some types of diode and other electronic device, and (c) as the emission mechanism for cold-cathode electron sources. Clearer theory may be helpful. We start by defining the theoretical context.

In this letter, $a\left(=1.541434 \times 10^{-6} \mathrm{~A} \mathrm{eV} \mathrm{V}^{-2}\right)$ and $b$ $\left(=6.830890 \mathrm{eV}^{-3 / 2} \mathrm{~V} \mathrm{~nm}^{-1}\right)$ are the first and second FowlerNordheim constants, $\varepsilon_{0}$ is the electric constant, and $\phi$ is the local work function of the emitting surface. $F$ denotes the barrier field and $J$ the emission current density; in CFE theory these positive quantities are the negative of the likenamed quantities used in conventional electrostatics. The "unreduced height" $h$ of a barrier is its height when the applied field is zero.

In early 1928, Fowler and Nordheim ${ }^{1}$ (FN) published their seminal paper on CFE theory, but knew their triangular tunneling barrier was physically unrealistic. Schottky's image-rounded barrier ${ }^{2}$ is better, but for this barrier the Schrödinger equation has no exact analytical solutions. So in late 1928 Nordheim $^{3}$ used the simple Jeffreys-WentzelKramers-Brillouin (JWKB) approximation ${ }^{4,5}$ to calculate values for a correction factor to the FN exponent (analogous to $v_{F}$ below). A mathematical error was found later. ${ }^{6}$ The equation now called the "standard Fowler-Nordheim equation" is the zero-temperature version of an equation derived by Murphy and Good $^{7}$ in 1956, again using a JWKB-type approximation. This "standard FN equation" is

$$
J=t_{F}^{-2} a \phi^{-1} F^{2} \exp \left\{-v_{F} b \phi^{3 / 2} / F\right\},
$$

where $v_{F}$ and $t_{F}$ are the values, for $h=\phi$, of the special field emission elliptic functions $v$ and $t$ defined in Ref. 7. (Reference 8 may be easier to follow. ${ }^{9}$ )

The functions $v$ and $t$ have purely mathematical definitions that depend only on a single variable. In CFE theory

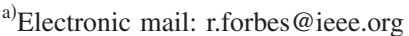

this has been the Nordheim parameter $y$ defined by

$$
y=c F^{1 / 2} / h
$$

where $c \equiv\left(e^{3} / 4 \pi \varepsilon_{0}\right)^{1 / 2}=1.199985 \mathrm{eV} \mathrm{V}^{-1 / 2} \mathrm{~nm}^{1 / 2} . t$ can be expressed in terms of $v$ and $d v / d y$ by

$$
t(y)=v-(2 / 3) y d v / d y .
$$

FN-plot theory ${ }^{10}$ uses a slope correction factor $s$, an intercept correction factor $r$, and a related function $u$. $s$ and $u$ are special field emission elliptic functions that, like $t$, can be expressed in terms of $v$ and $d v / d y$. Equations (2)-(7) in Ref. 8 summarize the relevant mathematical definitions (but the subscript $N$ has been dropped here).

$v(y)$ can be expressed ${ }^{7,8}$ in terms of the complete elliptic integrals $K$ and $E$. These may be defined with argument the elliptic modulus $k$ or with argument the elliptic parameter $m$ $\left[=k^{2}\right]$. Using $m$ is the clearer convention. In terms of $K(m)$ and $E(m),{ }^{11}$ and for the range $0 \leqslant y \leqslant 1, v(y)$ is best written ${ }^{8}$ as

$$
v(y)=(1+y)^{1 / 2}[E(m)-y K(m)],
$$

where

$$
m=(1-y) /(1+y) \text {. }
$$

The special elliptic functions have been evaluated accurately by various methods, ${ }^{6,8,12,13}$ and the results tabulated. ${ }^{6,8,12-14}$ The most precise published tabulation of $s$, $t$, and $v$ is Miller's; ${ }^{13}$ Forbes and Jensen ${ }^{14}$ have tabulated $u$. However, there is also a need for algebraic formulas.

Nonautomated manipulation of formulas concerning complete elliptic integrals is very tiresome. As far as I know, Jensen and Ganguly ${ }^{15,16}$ are the only authors to have derived analytical approximations for $v(y)$. They obtained complex formulas that are awkward to substitute into the FN equation exponent.

Simple empirical formulas have been derived by fitting (e.g., Refs. 17-21). Typically, these work well over a limited range of $y$, but do not adequately capture the full mathematical behavior of $v(y)$. Several are poor approximations near $y=1$. 
TABLE I. Comparison of exact values of $v(y)$ with those given by Eq. (5).

\begin{tabular}{ccccc}
\hline \hline & $\begin{array}{c}v(y) \\
\text { exact }\end{array}$ & $\begin{array}{c}v(y) \\
\text { Eq. (5) }\end{array}$ & Error & $\begin{array}{c}\text { Percentage error } \\
(\%)\end{array}$ \\
\hline 0.0 & 1.00000 & 1.00000 & 0.00000 & $0.00 \%$ \\
0.1 & 0.98168 & 0.98232 & 0.00064 & $0.07 \%$ \\
0.2 & 0.93704 & 0.93854 & 0.00150 & $0.16 \%$ \\
0.3 & 0.87176 & 0.87388 & 0.00212 & $0.23 \%$ \\
0.4 & 0.78876 & 0.79113 & 0.00237 & $0.30 \%$ \\
0.5 & 0.68997 & 0.69224 & 0.00227 & $0.33 \%$ \\
0.6 & 0.57681 & 0.57870 & 0.00189 & $0.33 \%$ \\
0.7 & 0.45041 & 0.45174 & 0.00133 & $0.30 \%$ \\
0.8 & 0.31166 & 0.31240 & 0.00073 & $0.24 \%$ \\
0.9 & 0.16131 & 0.16155 & 0.00024 & $0.15 \%$ \\
1.0 & 0.00000 & 0.00000 & 0.00000 & $0.04 \%$ \\
\hline \hline
\end{tabular}

I report here the discovery of a simple, good working approximation for $v(y)$,

$$
v(y) \approx 1-y^{2}+(1 / 3) y^{2} \ln y .
$$

As shown in Table I, this formula predicts $v(y)$ to better than $0.33 \%$ over the whole range $0 \leqslant y \leqslant 1$. It was discovered empirically using a spreadsheet, ${ }^{8}$ after noting (1) that corrections to the known basic approximation $\left(1-y^{2}\right)$ need to go to zero at both $y=0$ and $y=1$, but the form $y^{n}\left(1-y^{n}\right)$ does not perform well, and (2) that initial attempts with the mathematical package MAPLE ${ }^{\mathrm{TM}}$ to solve the problem, although unsuccessful, generated terms of the form $y^{2 n} \ln y$. ( $n$ is a positive integer.)

A search for the mathematical basis of Eq. (5), with Dr. J. H. B. Deane, showed that MAPLE's elliptic functions use $k$ as their argument (we originally used $m$ ). So one enters the square root of the earlier expression for $m$. MAPLE ${ }^{\mathrm{TM}}$ then gives

$$
\begin{aligned}
v(y)= & 1-[(9 / 8) \ln 2+3 / 16] y^{2}+(3 / 8) y^{2} \ln y \\
& -[(27 / 256) \ln 2-(51 / 1024)] y^{4}+(9 / 256) y^{4} \ln y \\
& +O\left(y^{6}\right)+O\left(y^{6} \ln y\right), \\
\approx & 1-0.9673 y^{2}+0.3750 y^{2} \ln y-0.0233 y^{4} \\
& +0.0352 y^{4} \ln y+\cdots .
\end{aligned}
$$

The coefficients of the higher-order terms fall off relatively rapidly.

The form of Eq. (6) explains why an approximation of the form of Eq. (5) works. But it does not explain why taking the coefficient of the $y^{2} \ln y$ term equal to $1 / 3$ works as well as it does. The fit is very sensitive to this coefficient: using 0.34 improves the fit, but using 0.32 or 0.35 makes it noticeably worse. Series (6) might suggest taking it as 0.375 , but this choice is significantly worse than $1 / 3$.

In the upper part of the range $0 \leqslant y \leqslant 1$, Eq. (5) outperforms both Eq. (6b) and the three-term version of it. It seems that $1-y^{2}$ is a good approximation for the sum of the terms in Eq. (6) that do not involve $\ln y$, especially near $y=1$. [This sum lies between $\left(1-0.9673 y^{2}\right)$ near $y=0$ and $\left(1-y^{2}\right)$ near $y=1$.] We thus considered formulas of type

$$
v_{3}(y)=1-y^{2}+q y^{2} \ln y,
$$

where $q$ is an adjustable constant. where $q$ is an adjustable constant.
However, I believe that there are significant scientific advan-
Downloaded 30 Mar 2009 to 131.227.178.132. Redistribution subject to AIP license or copyright; see http://apl.aip.org/apl/copyright.jsp
It was originally thought that $q=1 / 3$ might be an exact result, derivable from an alternative expansion of $v(y)$, but we find no evidence of this. Rather, we now think that by happy accident $1 / 3$ is close to best-fit values for $q$. A best-fit value could be found in several ways, but existing literature ${ }^{20}$ quotes the maximum magnitude of the relative error (RE), here $\left\{v_{3}(y)-v(y)\right\} / v(y)$. By least-squares fitting, Deane ${ }^{22}$ found $q=0.3391$ and maximum RE magnitude of $0.31 \%$.

As an approximation for the whole range $0 \leqslant y \leqslant 1$, formula (5) performs better than all previously reported simple formulas. The next best is Miller's five-parameter formula, ${ }^{20}$ which has maximum RE magnitude of $0.50 \%$. So Eq. (5) may be useful numerically.

However, numerical accuracy is not the issue: accuracy in $v$ to about $2 \times 10^{-8}$ is easily achieved with the Hastings ${ }^{23}$ approximation formulas recorded in Ref. 11. The requirement is for algebraic simplicity coupled with accuracy sufficient for purpose (namely, easier mathematical analysis of the standard FN equation). Equation (5) satisfies this.

Equation (5) and the Ref. 8 definitions yield approximations for the other special elliptic functions,

$$
\begin{aligned}
& s(y) \approx 1-(1 / 6) y^{2}, \\
& t(y) \approx 1+(1 / 9)\left[y^{2}-y^{2} \ln y\right], \\
& u(y) \approx 5 / 6-(1 / 3) \ln y .
\end{aligned}
$$

For $s(y)$ and $t(y)$ these predict exact values to better than $0.4 \%$ for $0 \leqslant y \leqslant 1$; for $u(y)$ prediction is better than $0.5 \%$ for $0.1 \leqslant y \leqslant 1$ but deteriorates for lower $y$ values (this is not important in practice).

These formulas make some aspects of FN-plot behavior more obvious. For example, experimental data typically have $y^{2}$ within the range of $0.2-0.5$. Equation (8) shows that, over this range, the slope correction factor $s$ would vary only by about 5\%; so the FN-plot nonlinearity predicted by standard FN theory is difficult to detect experimentally.

The most important finding, however, is that the formulas for the special elliptic functions can be naturally rewritten in terms of the variable $f=y^{2}$. Thus, Eq. (5) becomes

$$
v(f) \approx 1-f+(1 / 6) f \ln f .
$$

When $h=\phi$, we have $f=F / F_{b}$, where $F_{b}$ is the field needed to reduce a barrier of unreduced height $\phi$ to zero $\left(F_{b}\right.$ was calculated by Schottky ${ }^{24}$ long ago). This result comes from Eq. (2), with $h=\phi$ : we call $f$ the "scaled barrier field."

It follows that the FN-exponent correction factor can be written as an explicit function of barrier field: when $h=\phi, v$ becomes $v_{F}$ and Eq. (11) becomes

$$
v_{F}(F)=1-\left(F / F_{b}\right)+(1 / 6)\left(F / F_{b}\right) \ln \left(F / F_{b}\right) .
$$

Thus, after nearly 80 years, and 50 years after Murphy and Good's formulation of CFE theory, we have a simple, good, explicit algebraic formula for the exponent correction factor in the standard FN equation. In future it will be much easier to analyze the mathematical behavior of this equation. But all useful formulas now in terms of $y$ will need reformulation in terms of $f$. A reworking of standard FN theory in greater depth, based on Eqs. (11) and (12), will be presented elsewhere. ${ }^{25}$

Clearly, for many years precise numerical values of the special elliptic functions have been available when needed. 
tages in this discovery of simple, good, approximate algebraic formulas that have a respectable mathematical origin and can easily be transformed to functions of barrier field.

There is the merit, important in an applied area, of making standard FN theory looks less obscure than hitherto. Second, these good approximation formulas should make aspects of existing theory easier to explain, in particular, the theory of FN plots. Third, in extending CFE theory, for example to curved emitters ${ }^{26}$ reliable approximate formulas for the correction factors that apply to planar geometry should be useful.

The author thanks Jonathan H. B. Deane for his assistance in using MAPLE ${ }^{\mathrm{TM}}$ and for useful discussions. The author also thanks C. J. Edgcombe for a preprint of Ref. 26: this stimulated my interest in approximations for $v(y)$.

${ }^{1}$ R. H. Fowler and L. W. Nordheim, Proc. R. Soc. London, Ser. A 119, 173 (1928).

${ }^{2}$ W. Schottky, Phys. Z. 15, 872 (1914)

${ }^{3}$ L. W. Nordheim, Proc. R. Soc. London, Ser. A 121, 626 (1928).

${ }^{4}$ H. J. Jeffreys, Proc. London Math. Soc. 23, 428 (1924).

${ }^{5}$ H. Fröman and P. O. Fröman, JWKB Approximation: Contributions to the Theory (North-Holland, Amsterdam, 1965).

${ }^{6}$ R. F. Burgess, H. Kroemer, and J. M. Houston, Phys. Rev. 90, 515 (1953).

${ }^{7}$ E. L. Murphy and R. H. Good, Phys. Rev. 102, 1464 (1956).
${ }^{8}$ R. G. Forbes, J. Vac. Sci. Technol. B 17, 534 (1999).

${ }^{9}$ In Ref. 8 typographic errors occur in the definition of $K(m)$ (both brackets should be raised to the power $-1 / 2$ ), and in coefficient $a_{4}$ in Eq. (32c) (its value should be $a_{4}=0.01451196212$ ).

${ }^{10}$ R. G. Forbes, J. Vac. Sci. Technol. B 17, 526 (1999).

${ }^{11}$ M. Abramowitz and I. A. Stegun, Handbook of Mathematical Functions (Dover, New York, 1965), Chap. 17, Formulas 17.3.34 and 17.3.36.

${ }^{12}$ R. H. Good and E. W. Müller, in Handbuch der Physik, edited by S. Flugge (Springer, Berlin, 1956), Vol. 21, p. 176.

${ }^{13}$ H. C. Miller, J. Franklin Inst. 282, 382 (1966).

${ }^{14}$ R. G. Forbes and K. L. Jensen, Ultramicroscopy 89, 17 (2001).

${ }^{15}$ K. L. Jensen and A. K. Ganguly, J. Vac. Sci. Technol. B 11, 371 (1993).

${ }^{16}$ K. L. Jensen, in Vacuum Microelectronics, edited by W. Zhu (Wiley, New York, 2001), Chap. 3, see p. 44.

${ }^{17}$ F. M. Charbonnier and E. E. Martin, J. Appl. Phys. 33, 1897 (1962).

${ }^{18}$ L. N. Dobretsov and M. V. Gomoyunova, Emission Electronics (Nauka, Moscow, 1966) (in Russian). Translated by Israel Programme for Scientific Translations, Jerusalem, 1971, see p. 302.

${ }^{19}$ C. A. Spindt, I. Brodie, L. Humphrey, and E. R. Westerberg, J. Appl. Phys. 47, 5248 (1976).

${ }^{20}$ H. C. Miller, J. Vac. Sci. Technol. 17, 691 (1980).

${ }^{21}$ P. W. Hawkes and E. Kasper, Principles of Electron Optics (Academic, London, 1989), Vol. 2, see p. 927.

${ }^{22}$ J. H. B. Deane (private communication).

${ }^{23}$ C. Hastings, Jr., Approximations for Digital Computers (Princeton University Press, Princeton, 1955).

${ }^{24}$ W. Schottky, Z. Phys. 14, 83 (1923).

${ }^{25}$ R. G. Forbes and J. H. B. Deane (unpublished).

${ }^{26}$ C. J. Edgcombe and N. de Jonge, J. Vac. Sci. Technol. B 42, 869 (2006). 